\title{
A UTILIZAÇÃO DA CHUPETA \\ E O DESENVOLVIMENTO SENSÓRIO MOTOR ORAL
}

\section{Pacifier's use and oral motor sensory development}

\author{
Cláudia Marina Tavares de Araújo ${ }^{(1)}$, Giselia Alves Pontes da Silva ${ }^{(2)}$, Sônia Bechara Coutinho ${ }^{(3)}$
}

\begin{abstract}
RESUMO
Objetivos: verificar parâmetros do desenvolvimento sensório motor oral de lactentes no terceiro mês de vida, com e sem o hábito de chupar chupeta, além de identificar a frequência do uso da chupeta entre crianças em aleitamento materno e crianças que iniciaram o desmame. Métodos: estudo observacional com corte transversal, envolvendo 74 bebês que nasceram e estavam realizando acompanhamento no Serviço de Puericultura de uma maternidade da rede pública, Recife-PE. Os dados foram obtidos por meio de entrevista com as mães e observação das crianças. Resultados: as que não utilizavam chupeta apresentaram frequentemente padrão postural global simétrico, melhores respostas aos reflexos orais e língua mais posteriorizada $(p=0,034)$. Conclusão: constatou-se que as crianças que não utilizaram chupeta apresentaram melhor postura das estruturas orais, como também melhores respostas em relação aos reflexos orais. Nas crianças em aleitamento materno exclusivo foi predominante o não uso da chupeta, havendo associação estatisticamente significante entre o aleitamento materno exclusivo e o não uso da chupeta $(p=0,001)$. Por outro lado, entre as crianças que já haviam iniciado o desmame, o uso da chupeta foi mais frequente.
\end{abstract}

DESCRITORES: Aleitamento Materno; Comportamento de Sucção; Desenvolvimento Infantil; Sistema Estomatognático

\section{INTRODUÇÃO}

A forma de alimentar o lactente está diretamente relacionada às habilidades motoras orais. Daí considerar-se o tipo de alimento - natural/artificial e a forma como é oferecido - peito/mamadeira, fatores determinantes no desenvolvimento motor oral e alimentar infantil ${ }^{1-7}$.

A substituição da amamentação natural por mamadeira pode desencadear prejuízos no desenvolvimento sensório motor oral, por falta da correta

(1) Fonoaudióloga; Professora Adjunta do Departamento de Fonoaudiologia do Centro de Ciências da Saúde da Universidade Federal de Pernambuco, UFPE, Recife, PE; Doutora em Nutrição do Programa de Pós-graduação em Nutrição da Universidade Federal de Pernambuco.

(2) Médica; Professora Associada do Departamento MaternoInfantil do Centro de Ciências da Saúde da Universidade Federal de Pernambuco, UFPE, Recife, PE; Doutora em Pediatria pela Universidade Federal de São Paulo.

(3) Médica; Professora Associada do Departamento MaternoInfantil do Centro de Ciências da Saúde da Universidade Federal de Pernambuco, UFPE, Recife, PE; Doutora em Nutrição do Programa de Pós-graduação em Nutrição da Universidade Federal de Pernambuco.

Conflito de interesse: INEXISTENTE estimulação das estruturas orofaciais, e favorecer a instalação de hábitos orais 1,2,8,9. Estes são caracterizados por padrões de contração muscular aprendidos, regulados por arcos reflexos. Os hábitos de sucção são ações adquiridas por repetições frequentes do movimento de sugar, destacando-se o uso da chupeta ${ }^{10,11}$.

Os hábitos orais têm sido amplamente estudados por profissionais de saúde por repercutir no desenvolvimento crânio-facial, comprometendo aspectos morfológicos e motores. A motricidade orofacial decorre da ação dos grupos musculares envolvidos, repercutindo principalmente nas funções como respiração, sucção, mastigação, deglutição e fonoarticulação, consideradas funções vitais ${ }^{2-4,8,10,12-14}$.

O processo de desenvolvimento funcional da alimentação, iniciado no primeiro mês de vida envolve integração sensoriomotora da deglutição com a respiração, coordenação mão-olho, adequação do tônus muscular e postura e maturação psicossocial ${ }^{9,10,12,15,16}$.

Por outro lado, há vários estudos sobre o desenvolvimento da sucção em neonatos, nos quais os critérios utilizados como rotina são anamnese e 
avaliação sensória motora oral, além da observação da sucção não nutritiva, nutritiva e da deglutição ${ }^{10,12}$.

Este artigo tem como objetivos verificar parâmetros do desenvolvimento sensório motor oral de lactentes no terceiro mês de vida, com e sem o hábito de chupar chupeta, além de identificar a frequência do uso da chupeta entre crianças em aleitamento materno e crianças que iniciaram o desmame.

\section{MÉTODOS}

Estudo observacional com corte transversal, considerando-se o desenvolvimento sensório motor oral das crianças que utilizavam chupeta, comparado ao desenvolvimento das crianças não expostas a este utensílio.

Desenvolvido na Maternidade Professor Bandeira Filho, "Hospital Amigo da Criança" desde 2002, conveniada ao Sistema Único de Saúde e pertencente ao Distrito Sanitário V, da Prefeitura da Cidade do Recife, Pernambuco.

A população foi constituída por 74 crianças com três meses de idade, acompanhadas no ambulatório de Puericultura daquela maternidade, nascidas a termo (idade gestacional ao nascimento igual ou maior que 37 semanas). Normalmente, houve registro do Capurro Somático. Não constando esse dado, seguiu-se, na ordem: data da última menstruação e ultra-sonografia; condições clínicas compatíveis com bons índices de vitalidade, ou seja, índice de Apgar igual ou maior que sete; peso ao nascer adequado para a idade gestacional. Além disso, foram critérios de inclusão ausência de malformações ou doenças que pudessem interferir no desenvolvimento psicomotor.

Os critérios de exclusão foram estabelecidos por fatores que pudessem prejudicar ou mesmo retardar o início da prática do aleitamento natural: transtornos clínicos neonatais e pós-natais; alterações genéticas e/ou neurológicas; más formações que interferissem na alimentação; problemas maternos físicos e/ou psíquicos que inviabilizassem a amamentação, como Síndrome da imunodeficiência adquirida, mastectomia e alterações mentais.

A avaliação fonoaudiológica aconteceu por meio de observação e manuseio das estruturas orofaciais, registrando-se detalhadamente aspectos referentes à postura habitual destas estruturas e às respostas da criança ao estímulo táctil.

Com o objetivo de melhor controlar as avaliações individuais realizadas, bem como ter material suficiente para comparação e análise, todas as crianças foram filmadas na postura de repouso e durante o manuseio.
Os dados sobre o desenvolvimento sensório motor oral e de alimentação foram registrados em protocolo pré-estabelecido. Cada criança teve seus dados registrados individualmente e por data de retorno, considerando a variação de dez dias antes ou depois da data do nascimento.

A equipe de coleta foi constituída por uma pesquisadora responsável e quatro estudantes do sexto período do curso de Fonoaudiologia. A pesquisadora responsável realizou treinamento específico para fins de coleta, explicando detalhadamente os aspectos questionados e avaliados. Foi realizado estudo piloto, com avaliação dos protocolos e desempenho da equipe.

O protocolo de avaliação foi elaborado pela pesquisadora, baseado na evolução do desenvolvimento normal de zero a três meses proposto pelo Conceito Neuroevolutivo - Bobath ${ }^{15}$ e por Alexander, Boheme e Cupps ${ }^{16}$.

Como variáveis de análise, foram considerados hábitos de sucção de chupeta e dedo, alimentação e o desenvolvimento sensório motor oral - controle cervical, postura habitual global, de lábios e língua, reações orais, respostas ao estímulo sensorial -, aos três meses de idade.

Os bebês foram inicialmente observados no colo da mãe e, em seguida, em mesa de superfície macia e avaliados nas posturas prono, supino e puxado para sentar. Neste momento, verificava-se controle cervical e postura global - padrão de movimento simétrico ou assimétrico.

Em relação aos aspectos sensório motor oral, foram observados: postura habitual dos lábios aberta, entreaberta e fechada; postura habitual da língua - anterior, entrearcos e posterior; resposta aos reflexos orais de procura, sucção e mordida; sensibilidade intra e extra-oral.

A investigação do reflexo de procura foi realizada por toque em região perioral, sendo resposta positiva o giro da cabeça em direção ao estímulo, seguido de abertura oral.

O reflexo de sucção foi avaliado por resposta à sucção rítmica diante de estímulo com dedo enluvado na porção anterior da língua e/ou palato duro.

A mordida fásica foi testada por meio de toque na região lateral da gengiva. A presença de movimentos rítmicos sequenciados de abertura e fechamento oral demonstrava resposta positiva.

Para investigação da sensibilidade intra e extraoral, foram realizados toques nas regiões da face e cavidade oral, utilizando mãos enluvadas ou brinquedo de borracha. Como comportamento de aproximação, teve-se expressão de interesse e satisfação; e de afastamento recusa ao estímulo.

Esta pesquisa atende ao Conselho Nacional de Saúde, Resolução №196/96 17, sendo previa- 
mente aprovada pelo Comitê de Ética em Pesquisa do Centro de Ciências da Saúde da Universidade Federal de Pernambuco, sob o nำ292/2005. Todos os participantes assinaram o Termo de Consentimento Livre e Esclarecido e consentiram a divulgação dos resultados.

Os dados colhidos foram digitados em dupla entrada, com checagem e validação de informação. O software utilizado na formatação do banco e na análise estatística foi o Epilnfo versão $6.04{ }^{18}$.

Os dados foram submetidos à análise estatística, envolvendo o teste Qui-quadrado de associação de Pearson. O nível de significância utilizado foi de $5,0 \%(p \leq 0,05)$.

\section{RESULTADOS}

A amostra foi constituída por 74 crianças com 90 dias de idade mais ou menos dez dias (90,0; DP6,9 dias) e suas mães. Houve discreto predomínio de crianças do sexo masculino $(54,1 \%)$. A idade das mães variou entre 15 e 40 anos (23,5; DP5,2 anos), havendo maior concentração de faixa etária entre 20 e 29 anos (63,5\%).

Com referência ao desenvolvimento global, 60 lactentes $(81,1 \%)$ mantinham postura assimétrica em relação ao corpo e 61 (82,4\%) não apresentavam controle cervical. Houve resposta positiva para reflexos orais de procura em 44 bebês $(59,5 \%)$ e de sucção em 71 (95,9\%) crianças. A mordida fásica foi observada em todos os participantes.

A postura habitual de lábios aberta predominou em 71 crianças $(95,9 \%)$, assim como a língua projetada em 72 (97,3\%). Foram encontradas reações de afastamento em 63 sujeitos $(85,1 \%)$.

Os dados da Tabela 1 revelam que houve diferença estatisticamente significante para postura habitual de língua ( $p=0,034)$ e o uso de chupeta, não sendo significante para os demais parâmetros de desenvolvimento global e sensório motor oral utilizados.

Para as crianças não usuárias de chupeta houve melhor resposta aos reflexos orais de procura e sucção e a língua espontaneamente estava mais posteriorizada.

Aos três meses de vida, 52 crianças (quase $70,0 \%$ ) estavam em aleitamento materno exclusivo. Das $22(30,0 \%)$ que iniciaram o desmame, $13(59,1 \%)$ o fizeram no primeiro mês de vida, com introdução da mamadeira.

O uso de chupeta esteve presente em 21 crianças $(28,4 \%)$, com início nos primeiros quinze dias de vida.

A Tabela 2 evidencia que das 52 crianças que estavam em aleitamento materno exclusivo aos três meses de idade, 43 (82,7\%) não usavam chupeta, enquanto nove $(17,3 \%)$ a utilizavam. A associação entre aleitamento materno exclusivo e uso da chupeta foi estatisticamente significante $(p=0,001)$. Esta tabela revela que, dentre as mães que ofereceram chupeta aos filhos, a maior frequência foi entre as mais jovens.

\section{DISCUSSÃO}

Neste estudo, as mães mais jovens foram as que mais ofereceram chupeta aos seus filhos. A idade materna constitui fator importante na prática do aleitamento, à medida que mães mais jovens tendem a desmamar seus filhos precocemente, bem como introduzir a chupeta, por influência de avós ou vizinhos ${ }^{19-22}$.

O hábito de usar chupeta prevaleceu em crianças que não estavam mais sendo exclusivamente amamentadas. Isto ratifica o que vem sendo descrito na literatura: a instalação de hábitos orais está frequentemente associada ao desmame, seja na qualidade de determinante ou como indicativo de dificuldades em manter a amamentação natural ${ }^{5,21-23}$.

Em relação à postura global, o padrão assimétrico e a ausência do controle cervical aos três meses foram prevalentes nas crianças participantes. Entretanto, nas que não utilizavam chupeta, registra-se com mais frequência a postura simétrica, apesar do mesmo não acontecer com relação ao controle cervical. É possível inferir que estes achados podem estar relacionados à idade das crianças, visto ser em torno dos três meses que tem início o controle cervical ${ }^{12,16}$.

Neste sentido, no terceiro mês de vida, a criança começa a assumir uma postura simétrica, demonstrando aumento de atividades orientadas para linha média corporal. O controle cervical e de ombros é iniciado por maior ativação da musculatura destas estruturas bilateralmente. A cabeça se posiciona mais na linha média por ação dos flexores contra a gravidade ${ }^{12,16}$.

Com referência aos reflexos orais de procura e sucção, não houve diferença significante entre os bebês usuários e não usuários de chupeta, encontrando melhor resposta, no entanto, neste último grupo.

A mordida fásica esteve presente em todas as crianças da amostra, constatando-se resposta normal nesta faixa etária. Esta é uma reação de defesa caracterizada por fechamento/abertura rítmica mandibular, em resposta ao estímulo tátil nas gengivas ${ }^{12,13}$.

A língua em postura habitual esteve mais posteriorizada na população sem chupeta, demonstrando maior maturação neste aspecto. Língua menos 
Tabela 1 - Avaliação do comportamento motor global e sensório motor oral segundo o uso da chupeta. Maternidade Professor Bandeira Filho. Recife - PE, 2006

\begin{tabular}{|c|c|c|c|c|c|c|c|}
\hline \multirow{3}{*}{ Variáveis } & \multicolumn{7}{|c|}{ Uso de Chupeta } \\
\hline & \multirow{2}{*}{$N=74$} & \multirow{2}{*}{$\%$} & \multicolumn{2}{|c|}{ Sim } & \multicolumn{2}{|c|}{ Não } & \multirow{2}{*}{ Valor de $p$} \\
\hline & & & $\mathrm{n}$ & $\%$ & $\mathrm{n}$ & $\%$ & \\
\hline \multicolumn{8}{|l|}{ Postura global } \\
\hline simétrica & 14 & 18,9 & 3 & 14,3 & 11 & 20,8 & $\mathrm{p}^{*}=0,744$ \\
\hline assimétrica & 60 & 81,1 & 18 & 85,7 & 42 & 79,2 & \\
\hline \multicolumn{8}{|c|}{ Controle cervical } \\
\hline Sim & 13 & 17,6 & 6 & 28,6 & 7 & 13,2 & $p^{*}=0,173$ \\
\hline Não & 61 & 82,4 & 15 & 71,4 & 46 & 86,8 & \\
\hline \multicolumn{8}{|c|}{ Reflexo de procura } \\
\hline Sim & 44 & 59,5 & 12 & 57,1 & 32 & 60,4 & $\mathrm{p}^{\dagger}=0,798$ \\
\hline Não & 30 & 40,5 & 9 & 42,9 & 21 & 39,6 & \\
\hline \multicolumn{8}{|c|}{ Reflexo de sucção } \\
\hline Sim & 71 & 95,9 & 20 & 95,2 & 51 & 96,2 & $p^{*}=1,000$ \\
\hline Não & 3 & 4,1 & 1 & 4,8 & 2 & 3,8 & \\
\hline \multicolumn{8}{|l|}{ Mordida fásica } \\
\hline Sim & 74 & 100 & 21 & 100 & 53 & 100 & $\ddagger$ \\
\hline Não & - & - & - & - & - & - & \\
\hline \multicolumn{8}{|l|}{ Postura lábios } \\
\hline aberta & 71 & 95,9 & 21 & 100 & 50 & 94,3 & $\mathrm{p}^{*}=0,554$ \\
\hline fechada & 3 & 4,1 & - & - & 3 & 5,7 & \\
\hline \multicolumn{8}{|c|}{ Postura de língua } \\
\hline anterior & 22 & 29,7 & 10 & 47,6 & 12 & 22,6 & $p^{*}=0,034^{\S}$ \\
\hline posterior & 52 & 70,3 & 11 & 52,4 & 41 & 77,4 & \\
\hline \multicolumn{8}{|c|}{ Reação de aproximação } \\
\hline Sim & 74 & 100 & 21 & 100 & 53 & 100 & $\ddagger$ \\
\hline Não & - & - & - & - & - & - & \\
\hline \multicolumn{8}{|c|}{ Reação de afastamento } \\
\hline Sim & 63 & 85,1 & 17 & 81 & 46 & 86,8 & $\mathrm{p}^{*}=0,495$ \\
\hline Não & 11 & 14,9 & 4 & 19 & 7 & 13,2 & \\
\hline
\end{tabular}

* Teste Exato de Fisher

† Teste Qui-quadrado de Pearson

‡ Não foi possível determinar devido à ocorrência de frequências nulas

$\S$ Associação significante a 5,0\%.

Tabela 2 - Uso de chupeta e as variáveis prática do aleitamento materno exclusivo e idade da mãe. Maternidade Professor Bandeira Filho. Recife - PE, 2006

\begin{tabular}{|c|c|c|c|c|c|c|c|}
\hline \multirow{3}{*}{ Variáveis } & \multicolumn{7}{|c|}{ Uso de Chupeta } \\
\hline & \multirow{2}{*}{$N=74$} & \multirow{2}{*}{$\%$} & \multicolumn{2}{|c|}{ Sim } & \multicolumn{2}{|c|}{ Não } & \multirow{2}{*}{ Valor de $p$} \\
\hline & & & $\mathrm{n}$ & $\%$ & $\mathrm{n}$ & $\%$ & \\
\hline \multicolumn{8}{|c|}{ Aleitamento materno exclusivo } \\
\hline Sim & 52 & 70,3 & 9 & 42,8 & 43 & 81,1 & $\mathrm{p}^{*}=0,001^{\dagger}$ \\
\hline Não & 22 & 29,7 & 12 & 57,2 & 10 & 18,5 & \\
\hline \multicolumn{8}{|c|}{ Faixa etária da mãe $e^{\ddagger}$} \\
\hline 15 a 19 anos & 15 & 20,5 & 5 & 23,8 & 10 & 19,2 & $p^{*}=0,806$ \\
\hline 20 a 29 anos & 47 & 64,4 & 14 & 66,7 & 33 & 63,4 & \\
\hline 30 a 39 anos & 11 & 15,1 & 2 & 9,5 & 9 & 17,3 & \\
\hline
\end{tabular}

* Teste Qui-quadrado de Pearson

${ }^{\dagger}$ Associação significante a 5,0\%

‡ Para uma pesquisada não havia a informação 
projetada apresenta maior mobilidade, facilita a formação e propulsão do bolo alimentar, além de permitir a introdução da alimentação complementar ${ }^{12}$.

$O$ aleitamento materno exclusivo aos três meses de idade predominou em não usuários de chupeta. Provavelmente, pelo fato de a coleta ocorrer em maternidade credenciada à "Iniciativa Hospital Amigo da Criança", caracterizada pela atenção ao aleitamento materno desde a assistência pré-natal, durante o período de internação hospitalar e seguimento na puericultura. No entanto, o uso da chupeta é uma prática cultural muito utilizada, mesmo em populações orientadas a não oferecê-la ${ }^{20,24}$.

Os primeiros meses de vida do lactente são marcados por mudanças nas relações anatômicas do corpo, incluindo-se cavidade oral, faringe e laringe. No desenvolvimento oral e no global, a precisão dos movimentos progride à medida que a habilidade de dissociação de movimentos é adquirida. Assim, a língua se move com a mandíbula, não sendo capaz de fazê-lo isoladamente ${ }^{10,12}$.

Com o crescimento da criança, acontecem alterações anatômicas e fisiológicas do sistema sensório motor oral. A mandíbula estende-se para baixo e para frente, aumentando o espaço intra-oral, o que favorece mudança do padrão de mobilidade das estruturas orais pelo fato de a língua estar mais posteriorizada, ou seja, melhor posicionada em cavidade oral. Por outro lado, há incremento da atividade labial com maior oclusão ${ }^{12,25}$.

A maturação normal da sucção e da deglutição pode ser verificada pela competência motora oral, organização neurológica e maturidade gastrintestinal, que dependem de fatores internos e externos à criança. Dos fatores internos, ressaltam-se saúde geral, experiência alimentar, habilidade para coordenar a respiração e prontidão para a alimentação. Constituem fatores externos, a quantidade e velocidade do fluxo de leite e o tipo de apoio oferecido pelas mães durante a alimentação ${ }^{10}$.

O desenvolvimento facial após o nascimento sofre interferência do trabalho muscular decorrente da sucção para extração do leite. Nesta atividade, a mandíbula realiza movimentos de abertura e fechamento, promovendo crescimento do terço anterior da face e prevenindo más oclusões. O controle motor oral evolui a partir da atividade muscular específica, na medida em que ocorre também evolução no desenvolvimento motor global. Como resultado, o bebê aceita novas experiências alimentares no tocante à consistência e textura, favorecendo o desenvolvimento sensório motor oral ${ }^{9,10}$.

A maioria dos estudos referentes às consequências de mamadeira e chupeta no desenvolvimento orofacial se dá com crianças maiores, a partir do primeiro ano. Estes trabalhos constatam alteração na oclusão labial por ação ineficiente da musculatura responsável e postura habitual atípica de língua, como sinais de hipotonia e protrusão. Indicam, ainda, alterações dearcos dentários com consequências em oclusão e articulação dos fonemas 4,14,26.

Assim, é possível inferir que a motricidade orofacial é beneficiada por meio do aleitamento materno, visto que envolve diversos músculos, o que não ocorre na alimentação por meio da mamadeira, quando o trabalho predominante é realizado pelos músculos bucinadores ${ }^{27}$. Acrescenta-se a esse aspecto a possibilidade de não saciar a necessidade de sucção dos bebês, que se tornam mais susceptíveis a desenvolverem hábitos de sucção de chupeta e/ou dedo. A prática do aleitamento materno exclusivo, então, pode minimizar a aquisição do hábito de chupeta, frequentemente encontrado em crianças que não mamaram ${ }^{26}$.

\section{CONCLUSÃO}

Em crianças não usuárias de chupeta, encontrou-se com maior frequência padrão motor global simétrico, melhor resposta aos reflexos orais de procura e sucção, postura de língua em posição mais posteriorizada.

Além disto, verificou-se que crianças aos três meses aleitadas exclusivamente no peito frequentemente não utilizam chupeta. Por outro lado, registra-se com maior frequência o uso de chupeta entre as que já haviam iniciado o desmame.

Apesar de esta pesquisa mostrar-se limitada por ser um corte transversal e conter amostra relativamente pequena, contribui para melhor compreensão do impacto da instalação de hábitos orais no desenvolvimento sensorial e motor de bebês. 


\section{ABSTRACT}

Purpose: to check the parameters of breastfed babies' oral motor sensory development in their third month of life, with and without the pacifier use and identify the periodicity of pacifier use amongst infants being exclusively breastfed and those who already started weaning. Methods: an observational study with transversal cut, involving 74 babies born at the Public Maternity, in Recife-PE, and being followed by the Child Welfare Service entity. Data were obtained through interviews with the mothers, followed by observation of the infants during their routine out-patient consultations. Results: in this study, the Infants who do not regularly use pacifier, showed standard symmetric global posture, better response to oral reflexes and more posteriorized tongue $(p=0.034)$. The non-usage of pacifier by exclusively breastfed children was predominant. Conclusion: it was verified that infants who did not use pacifier showed better oral structures posture, as well as better response in relation to oral reflexes. There was a statistically significant association between exclusive breastfeeding and the non-usage of pacifier $(p=0.001)$. On the other hand, among children in weaning, pacifier usage was more frequent.

KEYWORDS: Breast Feeding; Sucking Behavior; Child Development; Stomatognathic System

\section{REFERÊNCIAS}

1. Giugliani ERJ, Espírito Santo LC, Oliveira LD, Aerts D. Intake of water, herbal teas and non-breast milks during the first month of life: associated factors and impact on breastfeeding duration. Early Human Develop. 2008; 84(5):305-10.

2. Neiva FCB, Leone CR. Sucking in preterm newborns and the sucking stimulation. Pró-Fono. 2006; 18(2):141-50.

3. Val DC, Limongi SCO, Flabiano FC, Silva KCL. Sistema estomatognático e postura corporal na criança com alterações sensório-motoras. PróFono. 2005; 17(3):345-54.

4. Carrascoza KC, Possobon RF, Tomita LM, Moraes ABA. Consequences of bottle-feeding to the oral facial development of initially breastfed children. J Pediatr. 2006; 82(5):395-7.

5. França MCT, Giugliani ERJ, Oliveira LD, Weigert EML, Santo LCE, Köhler CV, Bonilha ALL. Uso de mamadeira no primeiro mês de vida: determinantes e influência na técnica de amamentação. Rev Saúde Pública 2008; 42(4):607-14.

6. Longo GZ, Souza JMP, Souza SB, Szarfarc SC. Crescimento de crianças até seis meses de idade, segundo categorias de aleitamento. Rev Bras Saúde Mater Infant. 2005; 5(1):109-18.

7. Marques RFSV, Lopez FA, Braga JAP. O crescimento de crianças alimentadas com leite materno exclusivo nos primeiros 6 meses de vida. $J$ Pediatr. 2004; 80(2):99-105.

8. Gomes CF, Trezza EMC, Murade ECM, Padovani CR. Surface electromyography of facial muscles during natural and artificial feeding of infants. $J$ Pediatr. 2006; 82(2):103-9.
9. Araújo CMT, Silva GAP. Alimentação complementar e desenvolvimento sensório motor oral: possíveis implicações. Temas sobre Desenv. 2005; 13(78):5-11.

10. Costa SP, van den Engel-Hoek L, Bos AF. Sucking and swallowing in infants and diagnostic tools. J Perinatol. 2008; 28(4):247-57.

11. Degan VV, Boni RC. Hábitos de sucção, mamadeira e chupeta. São José dos Campos: Pulso; 2004.

12. Rogers B, Arvedson J. Assessment of infant oral sensorimotor and swallowing function. Ment Retard Dev Disabil Res Rev. 2005; 11(1):74-82.

13. Gomes ICD, Proença MG, Limongi SCO. Avaliação e terapia da motricidade oral. In: Ferreira LP, Barros MCPP, Gomes ICD, Proença MG, Limongi SCO, Spinelli VP, et al. Temas de fonoaudiologia. 9. ed. São Paulo: Loyola; 2002. p. 59-119.

14. Oliveira AB, Souza FP, Chiappetta ALML. Relação entre hábitos de sucção não-nutritiva, tipo de aleitamento e má oclusões em crianças com dentição decídua. Rev CEFAC. 2006; 8(3):352-9.

15. The Bobath Centre. Notes to accompany the 8-week course in cerebral palsy. Londres: The Bobath Centre; 1993.

16. Alexander R, Boehme R, Cupps B. Normal development of functional motor skills: the first year of life. Tucson: Therapy Skill Builders; 1993.

17. Brasil. Ministério da Saúde. Conselho Nacional de Saúde. Resolução 196/96 do Conselho Nacional de Saúde/MS. Sobre diretrizes e normas regulamentadoras de pesquisa envolvendo seres humanos. [citado 2006 Nov 05]. Disponível em: URL:http://conselho.saude.gov.br/resolucoes/1996/ Reso196.doc. 
18. United States of America. Department of Health and Human Services. Centers of Disease Control Prevention (CDC). Translations Epilnfo. [cited 2006 Nov 05]. Disponível em: URL: http://www.cdc.gov/ Epilnfo/translations.htm\#Portuguese.

19. Bueno MB, Souza JMP, Souza SB, Paz SMRS, Gimeno SGA, Siqueira AAF. Riscos associados ao processo de desmame entre crianças nascidas em hospital universitário de São Paulo, entre 1998 e 1999: estudo de coorte prospectivo do primeiro ano de vida. Cad Saúde Pública. 2003; 19(5):1453-60. 20. Silveira FJF, Lamounier JA. Fatores associados à duração do aleitamento materno em três municípios na região do Alto Jequitinhonha, Minas Gerais, Brasil. Cad Saúde Pública. 2006; 22(1):69-77.

21. Araújo OD, Cunha AL, Lustosa LR, Nery IS, Mendonça RCM, Campelo SMA. Aleitamento materno: fatores que levam ao desmame precoce. Rev Bras Enferm. 2008; 61(4):488-92.

22. Alves CRL, Goulart EMA, Colosimo EA, Goulart LMHF. Fatores de risco para o desmame entre usuárias de uma unidade básica de saúde de Belo
Horizonte, Minas Gerais, Brasil, entre 1980 e 2004. Cad Saúde Pública. 2008; 24(6):1355-67. 23. Heringer MRC, Reis M, Pereira LFS, Di Ninno CQMS. A influência da amamentação natural no desenvolvimento dos hábitos orais. Rev CEFAC. 2005; 7(3):307-10.

24. Cunha AJLA, Leite AM, Machado MMT. Breastfeeding and pacifier use in Brazil. Indian $\mathrm{J}$ Pediatr. 2005; 72(3):209-12.

25. Araújo CMT, Silva GAP, Coutinho SB. Aleitamento materno e uso de chupeta: repercussões na alimentação e no desenvolvimento do sistema sensório motor oral. Rev Paul Pediatr. 2007; 25(1):59-65.

26. Carvalho GD. S.O.S. respirador bucal: uma visão funcional e clínica da amamentação. São Paulo: Lovise; 2003.

27. Zanini C, França MCT. Algumas considerações sobre o leite humano e aleitamento materno. In: Jacobi JS, Levy DS, Siva LMC. Disfagia: avaliação e tratamento. Rio de Janeiro: Revinter; 2003. p. 83-100.

RECEBIDO EM: 10/09/2008

ACEITO EM: 13/04/2009

Endereço para correspondência:

Cláudia Marina Tavares de Araújo

Rua Dr. Geraldo de Andrade, 75 ap. 801

Recife - PE

CEP: 52021-220

E-mail: claudiamarina@gmail.com 